Bull. Austral. Math. Soc.

Vol. 44 (1991) [467-476]

\title{
EXISTENCE OF SOLUTIONS OF NONLINEAR DIFFERENTIAL EQUATIONS WITH DEVIATING ARGUMENTS
}

\author{
K. Balachandran and S. Ilamaran
}

\begin{abstract}
We prove an existence theorem for nonlinear differential equations with deviating arguments and with implicit derivatives. The proof is based on the notion of measure of noncompactness and the Darbo fixed point theorem.
\end{abstract}

\section{INTRODUCTION}

The theory of differential equations with deviating arguments has been studied by several authors $[1,2,3,5,7,8,9]$. Banas [5] has proved an existence theorem for a differential equation with deviating arguments. Balachandran and Damaran [2, 3] proved existence theorems for nonlinear integral equations with deviating argument. In this paper we shall derive a set of sufficient conditions for the existence of a solution of differential equations with deviating arguments by using the measure of noncompactness.

Kuratowski [10] introduced the measure of noncompactness for the family of all bounded subsets of metric spaces defined by

$$
\begin{gathered}
\alpha(X)=\inf \{\varepsilon>0: X \text { can be covered with a finite number } \\
\text { of sets of diameter smaller than } \varepsilon\} .
\end{gathered}
$$

Another measure of noncompactness is the so-called ball measure (or Hausdorff measure). It is defined by the formula

$$
\begin{gathered}
\chi(X)=\inf \{\varepsilon>0: X \text { can be covered by a finite number of } \\
\text { balls of radii smaller than } \varepsilon\} .
\end{gathered}
$$

There are some other definitions of measure of noncompactness. In this paper we use the measure of noncompactness defined by Banas and Goebel [6].

Received 10 December 1990

Copyright Clearance Centre, Inc. Serial-fee code: 0004-9729/91 \$A2.00+0.00. 


\section{Notation and Definitions}

Let $(B,\|\cdot\|)$ be a fixed Banach space with the zero element 0 . Denote by $\mathcal{M}_{B}$ the family of all nonempty and bounded subsets of the space $B$, and by $\mathcal{N}_{B}$ the family of all nonempty and relatively compact subsets of $B$. For a nonempty family $Z$ of subsets of $B$, let $Z^{c}$ denote the subfamily of $Z$ consisting of all closed sets in $Z$. The closure of a set $X$ and its convex closure will be denoted by $\bar{X}$ and $\operatorname{Conv}(X)$ respectively.

Definition 1: A nonempty family $\mathcal{P} \subset \mathcal{N}_{B}$ will be called a kernel of a measure of noncompactness if it satisfies the following conditions:

1. $X \in \mathcal{P} \Rightarrow \bar{X} \in \mathcal{P}$;

2. $X \in \mathcal{P}, Y \subset X, Y \neq \emptyset \Rightarrow Y \in \mathcal{P}$;

3. $X \in \mathcal{P} \Rightarrow \operatorname{Conv}(X) \in \mathcal{P}$;

4. $X, Y \in \mathcal{P} \Rightarrow \lambda X+(1-\lambda) Y \in \mathcal{P}, \lambda \in[0,1]$;

5. $\mathcal{P}^{c}$ is a closed subspace of the space $\mathcal{M}_{B}^{c}$ in the topology generated by Hausdorff distance.

Definition 2: A function $\mu: \mathcal{M}_{B} \rightarrow[0, \infty)$ is said to be measure of noncompactness with kernel $\mathcal{P}$ if it satisfies the following conditions:

1. $\mu(X)=0 \Leftrightarrow X \in \mathcal{P}$;

2. $X \subset Y \Rightarrow \mu(X) \leqslant \mu(Y)$;

3. $\mu(X)=\mu(X)$;

4. $\mu(\operatorname{Conv} X)=\mu(X)$;

5. $\mu(\lambda X+(1-\lambda) Y) \leqslant \lambda \mu(X)+(1-\lambda) \mu(Y)$, for all $\lambda \in[0,1]$;

6. if $X_{n} \in \mathcal{M}_{B}^{\mathrm{c}}, X_{n+1} \subset X_{n}$, for $n=1,2, \ldots$ and if $\lim _{n \rightarrow \infty} \mu\left(X_{n}\right)=0$ then $\bigcap_{n=1}^{\infty} X_{n} \neq \emptyset$.

Definition 3: Let $M \subset B$ be a given nonempty set and let $T: M \rightarrow B$ be a continuous transformation such that $T X \in \mathcal{M}_{B}$ for any $X \in \mathcal{M}_{B}$. Let $\mu$ be a measure of noncompactness on the space $B$. A transformation $T$ will be called a $\mu$-contraction if there exists a constant $k \in[0,1)$ such that $\mu(T X) \leqslant k \mu(X)$ for each set $X \in \mathcal{M}_{B}$.

We prove our existence theorem by using the following modified version of the Darbo fixed point theorem [4].

THEOREM 1. Let $E$ be a nonempty, closed, convex and bounded subset of the space $B$ and let $T: E \rightarrow E$ be a $\mu$-contraction, where $\mu$ is an arbitrary measure of noncompactness. Then $T$ has at least one fixed point which belongs to kernel of $\mu$.

Let $p(t)$ be a given continuous function defined on the interval $[0, \infty)$ and taking real positive values. Let $C\left([0, \infty), p(t) ; R^{m}\right)$ be denoted by $C_{p}$, the set of all continuous 
functions from $[0, \infty)$ to $R^{m}$ such that

$$
\sup \{|x(t)| p(t): t \geqslant 0\}<\infty .
$$

Then $C_{p}$ forms a Banach space with respect to the norm.

$$
\|x\|=\sup \{|x(t)| p(t): t \geqslant 0\} .
$$

For our convenience, we shall introduce the following notation:

For every $x \in C_{p}, x \in \mathcal{M}_{C_{p}}, T>0$, and $\varepsilon>0$ we put

$$
\begin{aligned}
\omega^{T}(x, \varepsilon) & =\sup \{|x(t) p(t)-x(s) p(s)|: t, s \in[0, T],|t-s|<\varepsilon\}, \\
\omega^{T}(X, \varepsilon) & =\sup \left\{\omega^{T}(x, \varepsilon): x \in X\right\} \\
\omega_{0}^{T}(X) & =\lim _{\varepsilon \rightarrow 0} \omega^{T}(X, \varepsilon), \\
\omega_{0}(X) & =\lim _{T \rightarrow \infty} \omega_{0}^{T}(X), \\
\gamma^{T}(x, \varepsilon) & =\sup \{|x(t)-x(s)|:|t-s| \leqslant \varepsilon, t, s \in[0, T]\}, \\
a(X) & =\lim _{T \rightarrow \infty} \sup _{x \in X}\{\sup [|x(t)| p(t): t \geqslant T]\}, \\
\mu(X) & =\omega_{0}(X)+a(X) .
\end{aligned}
$$

The function $\mu(X)$, defined by the last formula, is the measure of noncompactness in the space $C_{p}[6]$.

\section{BASIC ASSUMPTIONS}

Consider the differential equation of the form

$$
x^{\prime}(t)=f\left(t, x\left(H_{1}(t)\right), \ldots, x\left(H_{n}(t)\right), x^{\prime}\left(h_{1}(t)\right), \ldots x^{\prime}\left(h_{n}(t)\right)\right)
$$

with the initial condition

$$
x(0)=0
$$

where $x(t)$ is an unknown function.

Assume the following conditions:

(i) The functions $H_{i}, h_{i}:[0, \infty) \rightarrow[0, \infty)$ are continuous.

(ii) The function $f:[0, \infty) \times R^{m n} \times R^{m n} \rightarrow R^{m}$ is continuous.

If we define $x^{\prime}(t)=y(t)$, then the equation (1) with condition (2) will be transformed into the following functional-integral equation:

$$
y(t)=f\left(t, \int_{0}^{H_{1}(t)} y(s) d s, \ldots, \int_{0}^{H_{n}(t)} y(s) d s, y\left(h_{1}(t)\right), \ldots, y\left(h_{n}(t)\right)\right)
$$

$$
t \geqslant 0
$$


(iii) The function $f\left(t, x_{1}, \ldots, x_{n}, y_{1}, \ldots, y_{n}\right)$ satisfies the condition

$$
\begin{aligned}
& \left|f\left(t, x_{1}, \ldots, x_{n}, y_{1}, \ldots, y_{n}\right)-f\left(t, x_{1}, \ldots, x_{n}, z_{1}, \ldots, z_{n}\right)\right| \\
& \leqslant k_{1} \sum_{i=1}^{n}\left|y_{i}-z_{i}\right|, \quad k_{1} \geqslant 0 .
\end{aligned}
$$

(iv) $\left|f\left(t, x_{1}, \ldots, x_{n}, 0, \ldots, 0\right)\right| \leqslant L_{0}(t)+e^{L_{1}(t)} \sum_{i=1}^{n}\left|x_{i}\right|$, where $L_{0}:[0, \infty) \rightarrow[0, \infty)$ is a continuous function such that $\lim _{t \rightarrow \infty} L_{0}(t) \exp \left(-\int_{0}^{t} L_{0}(s) d s\right)=0$ and $L_{1}:[0, \infty) \rightarrow R$ is a continuous decreasing function such that $\lim _{t \rightarrow \infty} t e^{L_{1}(t)}=0$.

(v) $H_{i}(t) \geqslant t, \lim _{t \rightarrow \infty}\left(H_{i}(t)-t\right)=0$ and

$$
\sup \left\{\int_{t}^{H_{i}(t)} L_{0}(s) d s: t \geqslant 0\right\}<\infty \quad \text { for } i=1, \ldots, n .
$$

(vi) $h_{i}(t) \leqslant t$ and $\lim _{t \rightarrow \infty}\left(t-h_{i}(t)\right)=0$ for $i=1, \ldots, n$.

For every $t \geqslant 0$ define

$$
L(t)=\int_{0}^{t}\left[L_{0}(s)+e^{L_{1}(s)}\right] d s .
$$

Take an arbitrary number $M>1$ and consider the space $C_{p}$ with $p(t)=$ $e^{-M L(t)-t}$.

From (iv) and (v) the number

$$
m_{i}=\sup \left\{\left(e^{L_{1}(t)} \int_{t}^{H_{i}(t)} e^{M L(s)} d s\right) e^{-M L(t)}\right\}<\infty .
$$

(vii) assume that $k=n k_{1}+k_{2}+(n / M)<1$ where $k_{2}=\sum_{i=1}^{n} m_{i}$.

\section{EXISTENCE ThEOREM}

ThEOREM 2. Assume that the hypotheses (i) to (vii) hold; then the equation (3) has at least one solution $y(t)$ in $C_{p}$ such that $|y(t)| \leqslant r e^{M L(t)}$ where $r=(1-k)^{-1}$ $\sup \left\{L_{0}(t) e^{-M L(t)}: t \geqslant 0\right\}$

Proof: Define a transformation $F$ in the space $C_{p}$ by

$$
(F y)(t)=f\left(t, \int_{0}^{H_{1}(t)} y(s) d s, \ldots, \int_{0}^{H_{n}(t)} y(s) d s, y\left(h_{1}(t)\right), \ldots, y\left(h_{n}(t)\right)\right), t \geqslant 0
$$

and a set $E$ by

$$
E=\left\{y \in C_{p}:|y(t)| \leqslant r e^{M L(t)}\right\}
$$


where $r=(1-k)^{-1} \sup \left\{L_{0}(t) e^{-M L(t)}: t \geqslant 0\right\}$.

Clearly $E$ is nonempty, bounded, convex and closed in $C_{p}$. Now we prove that $F$ maps the set $E$ into itself. Take $y \in E$. Then from our assumptions we have

$$
\begin{aligned}
& |(F y)(t)| e^{-M L(t)} \\
& \leqslant \mid f\left(t, \int_{0}^{H_{1}(t)} y(s) d s, \ldots, \int_{0}^{H_{n}(t)} y(s) d s, y\left(h_{1}(t)\right), \ldots, y\left(h_{n}(t)\right)\right) \\
& -f\left(t, \int_{0}^{H_{1}(t)} y(s) d s, \ldots, \int_{0}^{H_{n}(t)} y(s) d s, 0, \ldots, 0\right) \mid e^{-M L(t)} \\
& +\left|f\left(t, \int_{0}^{H_{1}(t)} y(s) d s, \ldots, \int_{0}^{H_{n}(t)} y(s) d s, 0, \ldots, 0\right)\right| e^{-M L(t)} \\
& \leqslant k_{1} \sum_{i=1}^{n}\left|y\left(h_{i}(t)\right)\right| e^{-M L(t)}+\left[L_{0}(t)+e^{L_{1}(t)} \sum_{i=1}^{n} \int_{0}^{H_{i}(t)}|y(s)| d s\right] e^{-M L(t)} \\
& \leqslant k_{1} \sum_{i=1}^{n} r e^{M L\left(h_{i}(t)\right)} e^{-M L(t)}+L_{0}(t) e^{-M L(t)}+\left[e^{L_{1}(t)} \sum_{i=1}^{n} \int_{0}^{t}|y(s)| d s\right] e^{-M L(t)} \\
& +\left[e^{L_{1}(t)} \sum_{i=1}^{n} \int_{t}^{H_{i}(t)}|y(s)| d s\right] e^{-M L(t)} \\
& \leqslant k_{1} n r+r(1-k)+n r\left[e^{L_{1}(t)} \int_{0}^{t} e^{M L(\varepsilon)} d s\right] e^{-M L(t)} \\
& +r \sum_{i=1}^{n}\left[e^{L_{1}(t)} \int_{t}^{H_{i}(t)} e^{M L(s)} d s\right] e^{-M L(t)} \\
& \leqslant k_{1} n r+r(1-k)+r(n / M)\left[\int_{0}^{t} M\left(L_{0}(s)+e^{L_{1}(o)}\right) e^{M L(0)} d s\right] e^{-M L(t)}+r \sum_{i=1}^{n} m_{i} \\
& \leqslant k_{1} n r+r(1-k)+r(n / M)+r k_{2} \\
& =r \text {. }
\end{aligned}
$$

From this we observe that $F E \subset E$.

Now we prove that $F$ is continuous on the set $E$. For this let us fix $\varepsilon>0$ and $y, z \in E$ such that $\|y-z\|<\varepsilon$. From our assumptions we have

$$
\begin{aligned}
& |(F y)(t)-(F z)(t)| e^{-M L(t)-t} \\
& \quad \leqslant \mid f\left(t, \int_{0}^{H_{1}(t)} y(s) d s, \ldots, \int_{0}^{H_{n}(t)} y(s) d s, y\left(h_{1}(t)\right), \ldots, y\left(h_{n}(t)\right)\right)
\end{aligned}
$$




$$
\begin{aligned}
& -f\left(t, \int_{0}^{H_{1}(t)} y(s) d s, \ldots, \int_{0}^{H_{n}(t)} y(s) d s, z\left(h_{1}(t)\right), \ldots, z\left(h_{n}(t)\right)\right) \mid e^{-M L(t)-t} \\
& +\mid f\left(t, \int_{0}^{H_{1}(t)} y(s) d s, \ldots, \int_{0}^{H_{n}(t)} y(s) d s, z\left(h_{1}(t)\right), \ldots, z\left(h_{n}(t)\right)\right) \\
& -f\left(t, \int_{0}^{H_{1}(t)} z(s) d s, \ldots, \int_{0}^{H_{n}(t)} z(s) d s, z\left(h_{1}(t)\right), \ldots, z\left(h_{n}(t)\right)\right) \mid e^{-M L(t)-t}
\end{aligned}
$$

(4)

$$
\begin{aligned}
\leqslant & k_{1} \sum_{i=1}^{n}\left|y\left(h_{i}(t)\right)-z\left(h_{i}(t)\right)\right| e^{-M L(t)-t} \\
& +\mid f\left(t, \int_{0}^{H_{1}(t)} y(s) d s, \ldots, \int_{0}^{H_{n}(t)} y(s) d s, z\left(h_{1}(t)\right), \ldots, z\left(h_{n}(t)\right)\right) \\
& -f\left(t, \int_{0}^{H_{1}(t)} z(s) d s, \ldots, \int_{0}^{H_{n}(t)} z(s) d s, z\left(h_{1}(t)\right), \ldots, z\left(h_{n}(t)\right)\right) \mid e^{-M L(t)-t} \\
\leqslant & k_{1}\|y-z\|+\mid f\left(t, \int_{0}^{H_{1}(t)} y(s) d s, \ldots, \int_{0}^{H_{n}(t)} y(s) d s, z\left(h_{1}(t)\right), \ldots, z\left(h_{n}(t)\right)\right) \\
& -f\left(t, \int_{0}^{H_{1}(t)} z(s) d s, \ldots, \int_{0}^{H_{n}(t)} z(s) d s, z\left(h_{1}(t)\right), \ldots, z\left(h_{n}(t)\right)\right) \mid e^{-M L(t)-t} .
\end{aligned}
$$

Take an arbitrary fixed $T>0$. In view of (ii) $f$ is uniformly continuous on the compact set

$$
\begin{aligned}
{[0, T] } & \times\left[-r N_{1} e^{M L\left(H_{1}(T)\right)}, r N_{1} e^{M L\left(H_{1}(T)\right)}\right]^{m} \times \ldots \\
& \times\left[-r N_{n} e^{M L\left(H_{n}(T)\right)}, r N_{n} e^{M L\left(H_{n}(T)\right)}\right]^{m} \times D^{m n}
\end{aligned}
$$

where $N_{i}=\max \left\{H_{i}(t): t \in[0, T]\right\}$ and $D=\left[-r e^{M L(T)}, r e^{M L(T)}\right]$. Therefore for every $t \in[0, T]$ we have

(5)

$$
\begin{aligned}
& \mid f\left(t, \int_{0}^{H_{1}(t)} y(s) d s, \ldots, \int_{0}^{H_{n}(t)} y(s) d s, z\left(h_{1}(t)\right), \ldots, z\left(h_{n}(t)\right)\right) \\
& \quad-f\left(t, \int_{0}^{H_{1}(t)} z(s) d s, \ldots, \int_{0}^{H_{n}(t)} z(s) d s, z\left(h_{1}(t)\right), \ldots, z\left(h_{n}(t)\right)\right) \mid e^{-M L(t)-t} \leqslant \varepsilon
\end{aligned}
$$


Further, let us take $t \geqslant T$. Then we have

$$
\begin{aligned}
|(F y)(t)-(F z)(t)| & \leqslant|(F y)(t)|+|(F z)(t)| \\
& \leqslant 2 r e^{M L(t)} \\
|(F y)(t)-(F z)(t)| p(t) & \leqslant 2 r e^{-t}
\end{aligned}
$$

Hence for sufficiently large $T$ we have

$$
|(F y)(t)-(F z)(t)| p(t) \leqslant \varepsilon
$$

From (4), (5) and (6) we observe that $F$ is continuous on $E$.

Take an arbitrary fixed $T>0, Y \subset E$ and $y \in Y$. From our assumptions, for an arbitrary $t \geqslant T$ we have

$$
\begin{aligned}
& |(F y)(t)| p(t) \leqslant k_{1} \sum_{i=1}^{n}\left|y\left(h_{i}(t)\right)\right| e^{-M L(t)-t} \\
& +\left[L_{0}(t)+e^{L_{1}(t)} \sum_{i=1}^{n} \int_{0}^{H_{i}(t)}|y(s)| d s\right] e^{-M L(t)-t} \\
& \leqslant k_{1} \sum_{i=1}^{n}\left|y\left(h_{i}(t)\right)\right| e^{-M L\left(h_{i}(t)\right)-h_{i}(t)}+L_{0}(t) e^{-M L(t)-t} \\
& +n T e^{L_{1}(T)} r+n\left[e^{L_{1}(t)} \int_{T}^{t}|y(s)| e^{-M L(s)-s} e^{M L(\bullet)} d s\right] e^{-M L(t)} \\
& +e^{L_{1}(t)} \sum_{i=1}^{n}\left[\int_{t}^{H_{i}(t)} r e^{M L(s)} d s\right] e^{-M L(t)-t} \\
& \leqslant k_{1} \sum_{i=1}^{n}\left|y\left(h_{i}(t)\right)\right| e^{-M L\left(h_{i}(t)\right)-h_{i}(t)}+L_{0}(t) e^{-M L(t)-t} \\
& +n T e^{T_{1}(T)} r+\sup _{t \geqslant T}\left\{|y(t)| e^{-M L(t)-t}\right\} \\
& (n / M)\left[\int_{T}^{t} M\left(L_{0}(s)+e^{L_{1}(\bullet)}\right) e^{M L(\bullet)} d s\right] e^{-M L(t)} \\
& +\sum_{i=1}^{n} r e^{M L\left(H_{i}(t)\right)-M L(t)-t}\left(H_{i}(t)-t\right) e^{L_{1}(t)} \\
& \leqslant k_{1} \sum_{i=1}^{n}\left|y\left(h_{i}(t)\right)\right| e^{-M L\left(h_{i}(t)\right)-h_{i}(t)}+L_{0}(t) e^{-M L(t)-t} \\
& +n T e^{L_{1}(T)} r+(n / M) \sup _{t \geqslant T}\left\{|y(t)| e^{-M L(t)-t}\right\}
\end{aligned}
$$




$$
\begin{aligned}
& +r \sum_{i=1}^{n} e^{M\left\{L\left(H_{i}(t)\right)-L(t)\right\}-t}\left(H_{i}(t)-t\right) e^{L_{1}(t)} \\
\leqslant & {\left.\left[n k_{1}+(n / M)\right] \sup _{t \geqslant h(T)}\left\{|y(t)| e^{-M L(t)-t}\right\}+L_{0}(t) e^{-M L(t)-t}\right\} } \\
& +n T e^{L_{1}(T)} r+r \sum_{i=1}^{n} e^{M\left\{L\left(H_{i}(t)\right)-L(t)\right\}-t}\left(H_{i}(t)-t\right) e^{L_{1}(t)}
\end{aligned}
$$

From our assumptions we have

$$
\begin{aligned}
\lim _{t \rightarrow \infty} L_{0}(t) e^{-M L(t)-t}=\lim _{t \rightarrow \infty} t e^{L_{1}(t)} \\
=\lim _{t \rightarrow \infty} e^{M\left\{L\left(H_{i}(t)\right)-L(t)\right\}-t}\left(H_{i}(t)-t\right) e^{L_{1}(t)}=0
\end{aligned}
$$

and $\lim _{t \rightarrow \infty} h(t)=\infty$.

Therefore

$$
a(F Y) \leqslant\left(n k_{1}+(n / M)\right) a(Y) \leqslant k a(Y) .
$$

Fix $\varepsilon>0$ and $T>0$. Take $t, s \in[0, T]$ such that $|t-s| \leqslant \varepsilon$. Then for every $Y \subset E$ and for every $y \in Y$, we have

$$
\begin{aligned}
& \left|(F y)(t) e^{-M L(t)-t}-(F y)(s) e^{-M L(\bullet)-\bullet}\right| \\
& \leqslant\left|(F y)(t) e^{-M L(t)-t}-(F y)(t) e^{-M L(\bullet)-\bullet}\right| \\
& +\left|(F y)(t) e^{-M L(\bullet)-\bullet}-(F y)(s) e^{-M L(\bullet)-\bullet}\right| \\
& \leqslant\left|e^{-M L(t)-t}-e^{-M L(s)-\bullet}\right||(F y)(t)|+|(F y)(t)-(F y)(s)| \\
& \leqslant\left|e^{-M L(t)-t}-e^{-M L(\bullet)-\bullet}\right| \times \\
& \left\{\mid f\left(t, \int_{0}^{H_{1}(t)} y(s) d s, \ldots, \int_{0}^{H_{n}(t)} y(s) d s, y\left(h_{1}(t)\right), \ldots, y\left(h_{n}(t)\right)\right)\right. \\
& -f\left(s, \int_{0}^{H_{1}(t)} y(t) d t, \ldots, \int_{0}^{H_{n}(t)} y(t) d t, 0, \ldots, 0\right) \\
& \left.+\left|f\left(t, \int_{0}^{H_{1}(t)} y(s) d s, \ldots, \int_{0}^{H_{n}(t)} y(s) d s, 0, \ldots, 0\right)\right|\right\} \\
& +\mid f\left(t, \int_{0}^{H_{1}(t)} y(s) d s, \ldots, \int_{0}^{H_{n}(t)} y(s) d s, y\left(h_{1}(t)\right), \ldots, y\left(h_{n}(t)\right)\right)
\end{aligned}
$$




$$
\begin{aligned}
& -f\left(s, \int_{0}^{H_{1}(s)} y(\tau) d \tau, \ldots, \int_{0}^{H_{n}(s)} y(\tau) d \tau, y\left(h_{1}(s)\right), \ldots, y\left(h_{n}(s)\right)\right) \\
\leqslant & \gamma^{T}\left(e^{-M L(t)-t}, \varepsilon\right)\left[k_{1} r n e^{M L(T)}+\sup \left\{L_{0}(t): t \leqslant T\right\}+\right. \\
& \left.+r \sum_{i=1}^{n} \sup \left\{e^{L_{1}(t)} e^{M L\left(H_{i}(t)\right)} H_{i}(t): t \leqslant T\right\}\right]+\Delta^{T}(f, \varepsilon)
\end{aligned}
$$

where

$$
\begin{gathered}
\Delta^{T}(t, \varepsilon)=\sup \left[\left|f\left(t, x_{1}, \ldots, x_{n}, u_{1}, \ldots, u_{n}\right)-f\left(s, y_{1}, \ldots, y_{n}, v_{1}, \ldots, v_{n}\right)\right|:\right. \\
t, s \in[0, T],|t-s| \leqslant \varepsilon,\left|x_{i}-y_{i}\right| \leqslant r \gamma^{T}\left(H_{i}(t), \varepsilon\right) e^{M L\left(H_{i}(T)\right)}, \\
\left.\left|u_{i}-v_{i}\right| \leqslant 2 r e^{M L(t)}\right] .
\end{gathered}
$$

Thus $\omega_{0}^{T}(F Y)=0$ and consequently,

$$
\omega_{0}(F Y)=0
$$

Combining (7) and (8) we get

$$
\mu(F Y) \leqslant k \mu(Y)
$$

for any $Y \subset E$ so that $F$ is a $\mu$-contraction. Applying Theorem (1), we get a fixed point for $F$. This completes our existence theorem.

\section{REFERENCES}

[1] K. Balachandran, 'Existence of solution for nonlinear Volterra integral equation with deviating arguments', J. Math. Phys. Sci. 23 (1989), 201-206.

[2] K. Balachandran and S. Mamaran, 'An existence theorem for Volterra integral equation with deviating arguments', J. Appl. Math. and Stochastic Analysis 3 (1990), 155-162.

[3] K. Balachandran and S. Ilamaran, 'Existence of solution for nonlinear Volterra integral equations', Proc. Indian Acad. Sci. Math. Sci. 100 (1990), 179-184.

[4] J. Banas, 'On measures of noncompactness in Banach spaces', Comment. Math. Univ. Carolin. 21 (1980), 131-143.

[5] J. Banas, 'The existence and some properties of solutions of a differential equation with deviated argument', Comment. Math. Univ. Carolin. 2 (1981), 525-536.

[6] J. Banas and K. Goebel, Measures of noncompactness in Banach spaces: Lecture Notes in Pure and Applied Mathematics 60 (Marcel Dekker, Inc., New York and Basel, 1980).

[7] T.A. Burton, Volterra integral and differential equations (Academic Press, New York, 1983).

[8] S. Czerwik, 'The existence of global solutions of a functional-differential equation', Colloq. Math. 36 (1976), 121-125. 
[9] R.D. Driver, Ordinary and delay differential equation: Applied Math. Sciences 20, (Springer Verlag, Berlin, Heidelberg, New York, 1977).

[10] C. Kuratowski, 'Sur les espaces completes', Fund. Math. 15 (1930), 301-309.

Department of Mathematics

Bharathiar University

Coimbatore-641 046

India 